\title{
Le savoir-faire français en matière de lutte contre les pollutions marines accidentelles par hydrocarbures
}

\section{Rousseau}

Chargé des relations extérieures au centre de documentation, de recherche et d'expérimentations sur les pollutions accidentelles des eaux (CEDRE)

Résumé. - Victime de nombreuses pollutions par hydrocarbures le long de son littoral depuis les années 1970, la France a mis en place une organisation spécifique et originale, incluant notamment le Centre de Documentation, de Recherche et d'Expérimentations sur les pollutions accidentelles des eaux (CEDRE).

Le savoir faire français se caractérise par l'établissement de plans d'intervention (Plans Polmar, mer et terre) destinés à préparer et à coordonner la lutte contre les pollutions accidentelles des eaux en mer et sur le littoral.

Les opérations de lutte dépendent également de l'existence de moyens de lutte adaptés mis en œuvre par du personnel compétent.

Lors de pollutions à l'étranger, la France peut faire partager son expérience et son savoir-faire grâce à ses experts tels que ceux du CEDRE.

Depuis 13 ans déjà le CEDRE cherche, développe et teste les matériels et produits de lutte contre les pollutions marines accidentelles mais il s'intéresse également aux autres aspects de l'intervention que sont la détection des pollutions, leur suivi, leur modélisation, les banques de données, les systèmes d'aide à la décision, etc.

Les travaux poursuivis portent sur les actions de dépollution menées sur l'eau et sur le littoral et couvrent l'ensemble des techniques, de la collecte des hydrocarbures à l'élimination des déchets.

Mots-clés. - Hydrocarbures - Pollution - Accidentel - Mer - Littoral - France - CEDRE - Prévention - Navigation - Surveillance - Sécurité - Assistance - Plans d'intervention POLMAR - Lutte - Savoir-faire - Protection - Sites - Nettoyage - Restauration - Stockage - Élimination - Déchets.

Abstract. - As the victim of several oil spills caused by oil tanker accidents along its coasts during the 1970s, France has created a specific organization, including a research and documentation center, and developed all necessary means to prevent further major pollution and to protect its coastline.

In addition, French know-how is characterized by the establishment of intervention plans (POLMAR plans, sea and land) which are designed to prepare for and coordinate the 
fight against accidental water pollution at sea and on the coastline.

An antipollution operation also relies on the existence of the necessary means to carry it out, as well as specialized equipment which is ready to be put to work at sea as well as on the coastline by competent authorities. In the case of oil spills occurring in other countries, France is prepared to share its experience and know-how thanks to its groups of experts such as those of CEDRE.

Since 13 years CEDRE designs, develops and tests equipments and products to fight against accidental marine pollution. But he is also involved in other response aspect such as detection, monitoring, modeling, data bases, help to the decision systems. The range of CEDRE activities covers all the antipollution techniques from the collect of oil to wastes disposal.

La maritimisation de l'économie mondiale se poursuit régulièrement. Le tonnage de la flotte mondiale a été multiplié par 5 depuis 1948 et le tonnage transporté par 8 . En 1989, près de 4 milliards de tonnes de marchandises ont été transportés par mer dans le monde; sur le total, le pétrole et les produits pétroliers avec 1,47 milliards de tonnes représentent près de $38 \%$. Les statistiques nous apprennent encore que chaque tonne de produits pétroliers parcourt en moyenne 4800 milles marins $(9000 \mathrm{~km})$.

A l'accroissement du trafic maritime correspond un accroissement des risques d'accidents et de pollutions marines. Ceci a été vrai surtout dans les années 1970. A la fin de ces années, le volume global des pollutions provoquées par les pétroliers atteignait encore les 500000 tonnes; depuis, mise à part une forte poussée en
1983, les pollutions par pétroliers semblent être stabilisées autour de 80000 tonnes.

Quant à la France, elle n'échappe pas à la règle de la maritimisation croissante de son économie. En outre, sa position géographique la place en première ligne face aux risques causés par le trafic maritime européen. II convient de noter, en effet, que le tonnage du pétrole qui passe le long des côtes de France est en majorité destiné à des pays autres que la France et que les grandes pollutions qui ont affecté notre littoral ont été provoquées par des pétroliers à destination ou en provenance de ports autres que les ports français. C'est d'ailleurs cette série de pollutions accidentelles, surtout celle de l'Amoco Cadiz en 1978 (230000 tonnes, soit 6 fois celle de l'Exxon Valdez en Alaska) qui a conduit la France à renforcer sa politique de protection du milieu marin. Cette politique est basée sur trois piliers:

- la prévention,

- la préparation à la lutte,

- les opérations de lutte.

\section{PRÉVENTION}

La politique rigoureuse mise en place en 1978 s'appuie sur les réglementations internationales et européennes, renforcées par des mesures nationales et concerne essentiellement la sécurité et la surveillance de la navigation ainsi que l'intervention en mer. 


\section{I.1. LA SÉCURITÉ DE LA NAVIGATION}

II s'agit essentiellement de mesures destinées à:

- Renforcer le contrôle du respect des règles relatives à la construction, l'équipement et l'exploitation des navires: les centres de sécurité des Affaires Maritimes vérifient, au cours d'inspections, la conformité des navires aux règles de sécurité issues des conventions internationales, et notamment de la convention Marpol $73 / 78$. Le memorandum d'entente sur le contrôle des navires par l'Etat du port, signé à Paris le 26 janvier 1982, a permis d'harmoniser les contrôles des navires étrangers effectués dans les Etats européens signataires.

- Améliorer l'information des autorités sur le trafic maritime au large des côtes françaises: un décret du 24 mars 1978 institue un systeme de signalement des navires transportant des hydrocarbures lorsqu'ils pénètrent dans les eaux territoriales ou sont victimes d'une avarie à moins de 50 milles des côtes. Cette obligation se trouve désormais confortée par l'entrée en vigueur, depuis le 2 octobre 1983 du protocole I de la convention Marpol $73 / 78$, prévoyant que chaque événement entraînant ou pouvant entraîner le rejet en mer de substances nuisibles, fera l'objet d'un rapport aux autorités compétentes.

- Éloigner des côtes françaises la circulation des navires transportant des produits polluants: la circulation à moins de 7 milles des côtes des na- vires transportant des hydrocarbures a été interdite, sauf dans les chenaux d'accès à des ports et rades limitativement énumérés. A l'initiative de la France, le dispositif de séparation de trafic d'Ouessant a été modifié par l'Organisation Maritime Internationale (OMI) en janvier 1979 pour repousser le trafic des navires potentiellement dangereux à 27 milles des côtes.

- Aggraver les pénalités pour les infractions en matière de circulation maritime et de pollution des eaux de la mer.

\section{I.2. La surveillance de la navigation}

Les moyens nécessaires à l'application des mesures de prévention ont été renforcés.

Deux réseaux complémentaires permettent d'assurer cette mission:

- Cinq Centres Régionaux Opérationnels de Surveillance et de Sauvetage (CROSS), armés par du personnel militaire, coordonnent le sauvetage en mer, assurent la surveillance de la navigation maritime, notamment dans les dispositifs de séparation de trafic, et la surveillance des pollutions.

- Soixante cinq sémaphores de la Marine Nationale, assurent une veille optique et radar des côtes françaises. 


\section{I.3. L'INTERVENTION EN MER EN CAS DE MENACE DE POLLUTION}

Dans chaque région maritime, des moyens spécifiques ont été mis en place afin de pouvoir intervenir préventivement sur un navire en difficulté:

- Quatre remorqueurs civils d'intervention, de forte puissance, affrétés par la Marine Nationale et à la disposition des Préfets Maritimes, sont prêts en permanence à assister les navires en difficulté. Cette assistance peut être imposée par le Préfet Maritime s'il l'estime nécessaire.

- Des équipes d'évaluation et d'intervention peuvent être transportées par hélicoptère à bord de navires victimes d'avaries. Composées de militaires couvrant les différentes spécialités exercées à bord des navires, leur rôle est d'évaluer le danger, d'en informer le Préfet Maritime et éventuellement d'effectuer des interventions d'urgence à bord (préparation du remorquage, au besoin réparations de fortune), voire d'organiser l'évacuation du navire.

\section{PRÉPARATION À la LUTTE CONTRE LES POLLUTIONS MARINES ACCIDENTELLES}

L'efficacité de la politique nationale de prévention ne peut éliminer totalement le risque d'une pollution accidentelle.
Des efforts très importants ont donc été consacrés au renforcement du dispositif national de lutte contre la pollution dans ses différents aspects et notamment à la préparation à la lutte. Celle-ci repose essentiellement sur la planification des interventions et sur l'assistance que peuvent procurer des organisations spécialisées.

\section{II.1. La planification des interventions contre les pollutions accidentelles}

La première instruction organisant la lutte contre la pollution a été édictée en 1970 après l'accident du Torrey Canyon.

L'expérience tirée d'autres accidents a fait évoluer les conceptions concernant cette organisation qui a été modifiée intégralement dans une nouvelle instruction du 12 octobre 1978, organisant un plan Polmar, tenant compte notamment des enseignements de l'Amoco Cadiz.

L'organisation de la lutte contre les pollutions accidentelles est basée sur quelques principes de base:

\section{II.2. Distinction entre la lutte à terre et la lutte en mer}

- En mer, la responsabilité de préparer et de conduire les opérations de lutte incombe au Préfet Maritime, officier général de la Marine ayant d'importantes responsabilités militaires et civiles, et dépositaire de l'autorité de l'Etat en mer. 
- Dans chaque région maritime, le Préfet Maritime établit, en liaison avec les élus locaux et les usagers du milieu marin, un plan d'intervention en mer (Plan Polmar-Mer) qui recense notamment tous les moyens qui peuvent être affectés à la lutte.

- Dans chacun des 26 départements côtiers, le Préfet, représentant l'Etat dans le département, prépare un Plan Polmar-Terre en liaison avec les élus locaux, les usagers du milieu marin, les associations de protection de l'environnement et les experts scientifiques. Le plan Polmar-Terre doit comprendre un certain nombre d'éléments tels que le recensement des zones à protéger en priorité, les plans de pose des barrages destinés à protéger ces zones, l'inventaire des matériels de lutte publics et privés, la liste des sites de stockage et des centres de traitement des produits récupérés.

\subsection{Le recours à des organismes spécialisés}

Les interventions en mer et à terre font appel à des compétences spécialisées. Pour répondre à cette exigence, l'instruction Polmar a prévu la création du Centre de Documentation, de Recherches et d'Expérimentations sur les pollutions accidentelles des eaux (CEDRE).

Le CEDRE a pour missions principales l'amélioration des techniques de lutte contre la pollution, l'information des autorités concernées sur ces techniques et la formation du person- nel d'intervention. II assiste les administrations chargées de la préparation des plans d'intervention. En cas de pollution, il se tient à la disposition des autorités responsables de la lutte pour les conseiller en matière technique.

En outre, la Marine Nationale a créé pour répondre à ses propres besoins une Commission d'Etudes Pratiques Antipollution (CEPOL).

Enfin, le Ministère de l'Intérieur (Direction de la Défense et de la Sécurité Civile) possède, au sein des Unités d'Instruction de la Sécurité Civile (UISC), des sections d'intervention dotées de matériels spécifiques de lutte.

\section{OPÉRATIONS DE LUTTE}

Pour la conduite des opérations en mer et sur le littoral, Préfets Maritimes et Préfets disposent des équipements qui constituent les stocks POLMAR répartis sur le littoral.

La stratégie d'intervention consiste à déterminer le plus rapidement possible l'ampleur de la pollution et les principales caractéristiques des polluants afin d'adapter les moyens de lutte à la menace. Les renseignements nécessaires à cette première estimation peuvent être acquis par des opérations aériennes de télédétection. La Direction des Douanes possède un avion (bientôt deux) équipé à cet fin. 


\section{III.1. En mer}

Les opérations en mer consistent essentiellement à confiner et à récupérer le polluant et, dans certains cas à le traiter par des produits dispersants.

Dans la mesure du possible, le pétrole déversé en mer sera récupéré par des moyens mécaniques.

Les matériels existants ont été améliorés en vue de les intégrer dans un ensemble homogène et de les adapter aux moyens naval dont dispose la Marine Nationale. Des expérimentations réalisées en mer ont permis de définir des procédures opérationnelles de mise en œuvre.

Des citernes souples flottantes remorquables, d'une capacité de 50 à $100 \mathrm{~m}^{3}$, permettent le stockage et le transport des produits récupérés en mer. La vidange et le nettoyage de ces réservoirs ont été grandement facilités, notamment par l'adjonction d'un toit amovible à l'un des modèles existants.

Une autre approche s'efforce d'adapter des navires existants présentant de grandes capacités de stockage, petits pétroliers caboteurs ou dragues aspiratrices, comme supports de récupérateurs.

Enfin, d'autres techniques n'ont pas été négligées, notamment l'utilisation de barrages pour encercler une source localisée de pollution ou accroître la largeur balayée par un récupérateur, ou encore la mise au point de chaluts destinés à récupérer, à partir de petits navires de pêche, des nappes d'hydrocarbures visqueux ou traitées aux absorbants.

Bien que la récupération en mer soit généralement préconisée, des conditions météo-océaniques défavorables et l'urgence de la situation peuvent rendre nécessaire l'épandage de produits dispersants.

Les dispersants sélectionnés en France, présentent une faible toxicité, mais leur utilisation massive dans des zones confinées ou peu agitées risque de provoquer des conséquences dommageables pour l'environnement. Une limite géographique en mer a donc été déterminée au-delà de laquelle il est possible d'envisager leur utilisation massive sans risque majeur pour l'environnement.

Un programme d'expérimentations en mer a dégagé les données essentielles sur l'efficacité des moyens de traitement, le comportement du pétrole traité et sur les problèmes opérationnels. II est apparu en particulier que l'épandage correct des dispersants constitue un élément fondamental pour l'efficacité du traitement. Les matériels d'épandage existants ont été améliorés, et des équipements mieux adaptés au traitement par hélicoptère et à partir de navires ont été conçus.

Enfin, des expérimentations, menées en mer ou en laboratoire, ont permis d'évaluer d'autres produits de lutte: gélifiants, désémulsifiants, biodégradants, agents coulants. 


\section{III.2. En zone côtière et sur le littoral}

En zone côtière, la protection des sites sensibles définis dans les plans POLMAR locaux est une priorité.

La mise au point de spécifications techniques rigoureuses a permis aux autorités françaises de sélectionner les barrages les mieux adaptés. De nombreux exercices, réalisés régulièrement sur tout le littoral français, avec le concours du Service Technique des Phares et Balises, permettent à la fois de vérifier la validité des plans de pose et d'entraîner le personnel à la mise en œuvre des barrages de protection.

La pose de barrages côtiers est également conçue comme une technique destinée à confiner le pétrole en vue de le récupérer plus facilement. Les performances des récupérateurs côtiers ont été améliorées et des pompes adaptées aux produits visqueux ont été développées.

La recherche s'est également consacrée à la sélection des absorbants les plus efficaces, et à l'optimisation de leurs conditions d'utilisation, notamment en vue de protéger des zones écologiquement sensibles tels que les marais littoraux, où les moyens d'intervention sont très limités.

Quels que soient les moyens mis en œuvre pour traiter une pollution en mer, les expériences passées ont montré qu'une grande partie de la pollution finit toujours par toucher le littoral.
Pour ce qui concerne le nettoyage des plages, la priorité a été accordée à la mise au point de techniques de ramassage à la fois plus productives et plus sélectives permettant de limiter en amont les volumes de déchets à transporter et à traiter.

Les engins de travaux publics avaient été utilisés en grand nombre pour lutter contre les pollutions de l'Amoco Cadiz et du Tanio.

Des équipements spécifiques ont été conçus pour pouvoir être adaptés à ces engins et améliorer leurs performances.

L'efficacité du ramassage manuel a pu être améliorée grâce à la conception de matériels légers et très maniables, voire individuels, permettant de récupérer les hydrocarbures dans des zones difficilement accessibles.

La restauration des plages est désormais facilitée par l'emploi préalable de moyens de ramassage plus performants. De même, si la technique de nettoyage des rochers à l'aide de jets d'eau chaude sous pression reste utilisable, des produits de nettoyage plus efficaces et moins toxiques ont été sélectionnés.

Des bacs de stockage démontables et amovibles sont désormais associés aux chantiers de lutte. Un inventaire des sites potentiellement aptes à recevoir définitivement les déchets récupérés a été effectué au niveau national et intégré dans les plans d'intervention départementaux.

Les pollutions auxquelles les côtes françaises ont été soumises ont permis d'acquérir une solide expérience 
en matière de traitement des déchets pétroliers et en particulier de mettre au point le traitement à la chaux vive des résidus de type solide.

Parmi l'ensemble des réalisations figure la conception d'une installation mobile permettant le lavage sur place du sable et des galets pollués et leur restitution dans le milieu.

\section{FORMATION DES PERSONNELS ET EXERCICES ANTIPOLLUTION}

Dans un souci d'efficacité opérationnelle, les différents intervenants, en mer et à terre, se sont attachés à former les personnels qui participeront aux opérations de lutte.

Cette formation est, en règle générale, dispensée par le CEDRE qui dispose d'installations permettant d'organiser des stages pratiques. Bénéficient ainsi d'une formation adaptée, les personnels de la Marine Nationale, ceux du Ministère de la Mer qui gèrent et mettent en œuvre les équipements des stocks POLMARTERRE, ainsi que les corps de sapeurs-pompiers qui dans les départements constituent les équipes de première intervention des Préfets.

\section{CONCLUSION}

Les pollutions accidentelles majeures ont toujours un côté bénéfique: elle font progresser les connaissances et permettent ainsi d'accroître l'efficacité des contre-mesures.

Depuis 1967, il survient une pollution majeure tous les onze ans. En 1967, l'accident du Torrey-Canyon a servi de révélateur et a suscité la prise de conscience, par l'opinion publique internationale, du danger que représentaient les cargaisons de pétrole pour l'environnement marin.

Les premiers plans d'intervention voient le jour après cette catastrophe; les compagnies pétrolière suivies par les instances internationales, jettent les bases de l'indemnisation des dommages provoqués par les pollutions.

En 1978, la catastrophe de l'Amoco Cadiz survient alors que la Conférence sur le Droit de la Mer est réunie à Genève. A l'initiative de la délégation française, le texte de la Convention renforce les droits des Etats côtiers; en France, un certain nombre de dispositions sont prises pour améliorer la prévention. Les plans Polmar sont élaborés, le CEDRE est créé, les équipements et les techniques d'intervention progressent.

Les conséquences de l'échouement de l'Exxon Valdez en Alaska, en mars 1989, et de l'AGEAN SEA à la Corogne en décembre 1992, ne sont pas encore entièrement perceptibles. D'autres mesures seront certainement étudiées et prises, et la coopération internationale devrait en être renforcée.

Beaucoup a donc déjà été fait et les résultats sont encourageants; la diminution de la pollution des mers, même si elle s'explique partiellement 
par la réduction du trafic pétrolier à partir de 1974, doit être portée au crédit des mesures de prévention prises dans le monde. Beaucoup reste encore à faire, d'autant qu'à côté du risque pétrolier grandit un autre danger: le trafic des substances chimiques. C'est l'un des nouveaux domaines de recherches du CEDRE.

\section{BIBLIOGRAPHIE}

- Le savoir-faire français en matière de prévention et de lutte contre les pollutions accidentelles par hydrocarbures. Gilles Bergot (CEDRE, 1986).

- Le transport maritime français en 1989. Comité Central des Armateurs de France.

- La Sécurité des approvisionnements de l'Europe. (Colloque international de Brest, 1988). 